\title{
A numerical model for partially-wetted flow of thin liquid films
}

\author{
K. V. Meredith ${ }^{1}$, A. Heather ${ }^{2}$, J. de Vries ${ }^{1} \&$ Y. Xin ${ }^{1}$ \\ ${ }^{1}$ FM Global, Research Division, USA \\ ${ }^{2}$ OpenCFD, UK
}

\begin{abstract}
A model for simulating thin liquid film transport over solid surfaces has been developed. The film transport for mass continuity and momentum were formulated as a two-dimensional set of equations using thin-film assumptions. These equations have been implemented in an open-source CFD code (OpenFOAM). Treatments for partial-wetting phenomena have been included in the model to account for the behavior near the contact-line. A surface-tangential force along the contact line has been developed to allow for the simulation of rivulets and dry patches in two-dimensional surface flow. An approach for applying contact angle effects to the model for a real stochastic surface is outlined. Additionally, experimental measurements were made for film flow over an inclined surface for a wide range of flow rates. Using these experimental results, the model has been validated for partially wetted flow over an inclined panel. The critical flow rate of a film over a given surface was used as validation for the model. Results show that for flow rates below the critical flow rate, the partially wetted behavior of the flow was reproduced. Comparisons to experimental flow patterns and wetted-area fractions were made.
\end{abstract}

Keywords: thin-film assumption, partial wetting, contact line, critical flow rate.

\section{Introduction}

The goal of this research was to develop a robust water-film transport model for use in simulating the partial wetting behavior of thin film flow in practical applications over realistic surfaces. To this extent, a thin film transport model has been developed in OpenFOAM ${ }^{\circledR}$, an open source computational fluid dynamics (CFD) framework. 
Work on understanding thin-film flows began in 1910 with the experimental measurements of Hopf [1]. Later, Nusselt [2,3] proposed a theoretical treatment of thin-film flow and heat transfer. An excellent review of the work prior to 1964 has been offered by Fulford [4]. Recent film modeling has focused on volume of fluid (VOF) approaches [5,6] and on applying the lubrication approximation to thin-film flow [7-9]. The VOF models are computationally expensive. The majority of the lubrication approximation models are not aimed at practical problems, include a limited amount of additional physics, and are overly focused on detailed behavior near the contact line.

The phenomena that occur during the lifetime of a liquid film consist of partial wetted flow behavior and interfacial transport of mass, momentum, and energy. The subject of this work will focus on partially wetted flow behavior, i.e., surface film flows delineated by a contact line separating the dry and wet surfaces. In addition, this approach includes treatment for phenomena such as rivulets, dry patches, and isolated wet spots.

\section{Modeling approach}

Due to the thin nature of the liquid film (typically less than $1 \mathrm{~mm}$ ), the flow in the direction normal to the surface can reasonably be assumed to be negligible. The transport model only needs to describe film advection in the surface-tangential direction, thus greatly simplifying the model. In addition, due to the 'thinness' of the film, the diffusive transport of mass/momentum/energy in the surfacenormal direction will dominate and the surface-tangential diffusion will be small in comparison. In other words, advection can be treated in the wall-tangential direction and diffusion processes treated in the wall-normal direction. This is known as the thin-film assumption and has been used successfully by a number of authors [10-13]. Tangential diffusion effects may prove to be important along the contact line (e.g. at the edges of rivulets), and this feature may need to be added in the future.

The thin-film assumption allows integration of the three-dimensional transport equations in the wall-normal direction from the wall surface to the film surface to obtain a set of equations for the film thickness and the wall-tangential components of velocity.

\subsection{Mass continuity}

The integrated mass continuity equation is defined as

$$
\frac{\partial \rho \delta}{\partial t}+\nabla_{s} \cdot[\rho \delta \mathbf{U}]=S_{\rho \delta}
$$

where $\rho$ is the liquid density, $\delta$ is the film thickness, $\mathbf{U}$ is the film velocity, $\nabla_{s}$ is the vector differential operator tangential to the surface, $\left(\frac{\partial}{\partial x}, \frac{\partial}{\partial y}\right)^{\top} . S_{\rho \delta}$ is the mass source per unit wall area due to impingement, splashing, evaporation, absorption 
into the solid, and film separation. For the purposes of this study, where the focus was on film flow in the absence of spray interactions and phase change, the source term is reduced to

$$
S_{\rho \delta}=0
$$

\subsection{Momentum transport}

The momentum equation, integrated over film height, is

$$
\frac{\partial \rho \delta \mathbf{U}}{\partial t}+\nabla_{s} \cdot[\rho \delta \mathbf{U U}]=\underbrace{-\delta \nabla_{s} p}_{\text {pressure based }}+\underbrace{\mathbf{S}_{\rho \delta \mathbf{U}}}_{\text {stress based }}
$$

where $\mathbf{U}$ represents the mean, tangential velocity of the film. The momentum source terms are split into pressure based (tangential gradients in wall-normal forces) and stress based (forces tangential to wall).

The pressure term, $p$, is comprised of forces in the wall-normal direction and consists of capillary effects $\left(p_{\sigma}\right)$, hydrostatic pressure head $\left(p_{\delta}\right)$, and local gasphase pressure $\left(p_{g}\right)$.

$$
p=p_{\sigma}+p_{\delta}+p_{g}
$$

The stress term, $\mathbf{S}_{\rho \delta \mathbf{U}}$, includes the viscous shear stresses $\left(\boldsymbol{\tau}_{g}, \boldsymbol{\tau}_{w}\right)$, gravity body force $\left(\rho \mathbf{g}_{t} \delta\right)$, and stress related to contact-angle force $\left(\boldsymbol{\tau}_{\theta}\right)$.

$$
\mathbf{S}_{\rho \delta \mathbf{U}}=\boldsymbol{\tau}_{g}+\boldsymbol{\tau}_{w}+\rho \mathbf{g}_{t} \delta+\boldsymbol{\tau}_{\theta}
$$

The specific definitions and submodels for the pressure terms tangential momentum source terms in Equations 4 and 5 are given in Section 3.

\section{Source terms and submodels}

Relevant source terms in Equations (4) and (5) are described in detail. Where appropriate, a background section is included describing the theory behind the submodel for that particular source term.

\subsection{Capillary pressure}

The capillary pressure, $p_{\sigma}$, required by Equation (4), is the pressure component due to surface tension based on the curvature of the film surface and represented by the Laplace-Young boundary condition at the air-fluid interface $[7,14]$. Using the Laplacian of film thickness to estimate the curvature, the term for the pressure contribution of surface tension is given as

$$
p_{\sigma}=-\sigma \nabla_{s}^{2} \delta
$$

where $\sigma$ is the surface tension and $\nabla_{s}^{2} \delta$ approximates the curvature of the liquid surface. This relationship is valid only for surfaces with slight curvature [14]. 


\subsection{Hydrostatic pressure}

The hydrostatic pressure, $p_{\delta}$, required by Equation (4), is the pressure component due to hydrostatic pressure head, and is given by

$$
p_{\delta}=-\rho(\mathbf{n} \cdot \mathbf{g}) \delta
$$

where $\mathbf{g}$ is the gravity force vector and $\mathbf{n}$ is the surface normal vector. This pressure term is eliminated for purely vertical surfaces $(\mathbf{n} \cdot \mathbf{g}=0)$.

\subsection{Gravity body force}

The gravity body force term, required by Equation 5, is represented by $\rho \mathrm{g}_{t} \delta$ where $\mathbf{g}_{t}$ denotes the gravity components tangential to the wall. This term is zero for horizontal surfaces, and is the main driving force for flow over vertical and inclined surfaces.

\subsection{Shear stress}

The shear stress terms $\tau_{g}$ and $\tau_{w}$, required by Equation (5), represent the shear at the film-gas interface and the film-wall interface respectively.

In most cases for film flow in the presence of a quiescent gas, $\tau_{g} \ll \tau_{w}$ because gas-phase velocities are typically very low and also because the viscosity of the gas is low compared to that of the liquid. For the purposes of this study, $\tau_{g}$ is assumed negligible.

The film-wall stress term, $\tau_{w}$, is modeled based on an assumed velocity profile in the wall-normal direction. Assuming laminar flow and taking the velocity at the wall to be zero and the velocity gradient at the gas interface to be zero, the expression for the velocity distribution through the film can be shown to be

$$
\mathbf{u}(z)=\frac{3 \mathbf{U}}{\delta}\left(z-\frac{z^{2}}{2 \delta}\right)
$$

where $\mathbf{U}$ represents the mean film velocity, $z$ represents the surface-normal coordinate, and $\delta$ represents the film thickness. Employing the quadratic velocity profile assumption, the shear stress at the wall is found as

$$
\tau_{w}=-\mu \frac{3 \mathbf{U}}{\delta}
$$

Experimental data confirms that this parabolic velocity profile remains valid even for wavy film flows [15]. A film can exhibit wavy behavior without necessarily being turbulent, and is not fully turbulent until $\operatorname{Re}_{f}=\Gamma / \mu>900$ [4], which is well above the range of interest for this study. Here, $\Gamma$ is defined as the mass flow rate per unit width of inlet $(\mathrm{kg} / \mathrm{m}-\mathrm{s})$. 


\subsection{Partial wetting behavior}

Simulating partial wetting behavior involves treatment of the contact line (the delineation between wet/dry regions). Along the contact line delineating the separation of wet and dry regions of the flow there is a surface-tangential force that limits the film from spreading. This force only exists for partially wetting fluids (i.e. $\theta_{E}>0$, where $\theta_{E}$ represents the contact angle). The contact angle defines the ability of the liquid to flood the surface. This force is a major cause of partially wetted flow behavior such as rivulets, dry spots, and film rupture. Thus, the effect of contact angle must be included in the film transport model in order to accurately predict partial wetting behavior.

The tangential surface forces due to interfacial tension, $\left(\mathbf{F}_{s}^{\prime}\right)$, are expressed as

$$
\mathbf{F}_{s}^{\prime}=\left(\sigma+\sigma_{s l}\right)-\sigma_{s o}
$$

where $\sigma$ is the liquid-air interfacial tension, $\sigma_{s l}$ is the solid-liquid interfacial tension, and $\sigma_{s o}$ is the solid-air interfacial tension.

Since only $\sigma$ in Equation (10) is easily measured experimentally, it is helpful to reduce this surface-tangential force to a function of $\sigma$ and $\theta_{E}$ (also a measurable quantity) by employing Young's Law $[16,17]$. Young's Law states that the forces along the contact line are balanced at equilibrium as

$$
\sigma_{s o}-\left(\sigma \cos \theta_{E}+\sigma_{s l}\right)=0
$$

Combining Equations (10) and (11) yields what is termed here as the 'contactangle force'.

$$
\mathbf{F}_{s}^{\prime}=\sigma\left(1-\cos \theta_{E}\right)
$$

$\mathbf{F}_{s}^{\prime}$ represents the surface-tangential force normal to the contact line (still in the tangential plane) per unit width of contact line.

The contact-angle force is applied in a straight forward fashion to the momentum equation, as is shown here:

$$
\boldsymbol{\tau}_{\theta}=\beta \frac{\sigma\left(1-\cos \theta_{E}\right)}{\Delta_{c l}} \mathbf{n}_{c l}
$$

First, $\mathbf{F}_{s}^{\prime}$ is divided by the width of the computational cell, $\Delta_{c l}$ in the direction normal to the contact line. Secondly, $\mathbf{F}_{s}^{\prime}$ is multiplied by the unit vector normal to the contact line in the surface-tangential plane. Finally, an empirical parameter $\beta$ is introduced to account for discrepancies between the theory and observed behavior of real surfaces. Values for $\beta$ can be determined by experimentally measuring the critical flow rate (i.e. the flow rate at which transition occurs from rivulet flow to continuous film flow occurs) for a given surface, and then adjusting $\beta$ to match this behavior in the model. 


\subsection{Specification of contact line}

The contact line is defined as the interface between the wet regions and dry regions in the computation. In this context, it is helpful to think in terms of a critical film thickness. For a given contact angle, a film will exhibit a minimum or critical film thickness, $\delta_{c}$, below which the film flow will not exert enough force to overcome the contact angle force. Analytical expressions can be derived for $\delta_{c}$ on horizontal, vertical, and arbitrary inclination angle surfaces. In the model, a region of the flow is considered wet when denoted by an $\omega$ value of 1 , and considered dry when denoted by an $\omega$ value of 0 . Determination of $\omega$ depends on the critical film thickness and the approach varies for hydrophobic surfaces versus hydrophilic surfaces.

Hydrophobic surfaces For surfaces that are non-absorbent of water, when $\delta>$ $\delta_{c}$, then that region of the film is considered 'wet', while for $\delta<\delta_{c}$ the surface is considered 'dry'.

Hydrophilic surfaces For certain porous materials (such as corrugated cardboard) or surfaces with micro-grooves that tend to attract water, once the surface has been 'wetted' it is very difficult to return it to the 'dry' state, even when $\delta<\delta_{c}$. To account for this in the model, a 'memory' is introduced that records whether that region of the surface was previously wetted. If a region of the film was previously wetted, then $\delta$ must reach a value much lower than $\delta_{c}$ (e.g. $\delta<0.001 \delta_{c}$ ) in order to be considered dry again.

To account for the scenario where the surface is wetted by means other than film flow (e.g. spray impingement) and the film thickness is still lower than $\delta_{c}$, the wetting criterion has been relaxed to be, for example, $\delta>\frac{1}{2} \delta_{c}$.

In practice, this phenomenologically based treatment does a very acceptable job of replicating experimentally observed partial wetting behavior.

\subsection{Specification of contact angle}

One of the major issues with the above approaches is determining the contact angle. The contact angle for advancing films or flowing rivulets has usually been found to be different than equilibrium contact angles measured from the droplet method $[15,18]$. The contact angle has even been found to be dependent on flow rate, rivulet width, and heat transfer rate [19]. For water on cast acrylic (Plexiglas ${ }^{\circledR}$ ), the surface used in this study, the contact angle has been observed by the authors to typically be in the range of $70^{\circ}$. Thus, in the model a normal distribution of contact angle is used based on an experimentally observed mean value and standard deviation. For the results shown here, the standard deviation was chosen as $10^{\circ}$ with a mean of $75^{\circ}$. At each computational cell a random contact angle is chosen from this normal distribution and is fixed for the duration of the calculation. The result of this approach is a contact angle that varies over the 
surface in a random manner. This gives rise to nonuniform flow and leads to the formation of rivulets and dry regions.

\section{Experimental setup}

The experiments used a test apparatus specially designed to generate uniform water flows. The main body of the apparatus consists of a vertical cast acrylic plate $(0.61 \mathrm{~m}$ wide and $1.22 \mathrm{~m}$ long). On the upper end of the plate, a perforated copper tube $(1.27 \mathrm{~cm}$ diameter, $0.51 \mathrm{~m}$ long) was positioned laterally to discharge water through sixty holes (1 $\mathrm{mm}$ diameter) onto the cast acrylic panel. Since the discharge of the individual stream created wavy flow, the water discharged from tube applicator was forced through a diffusive medium attached to the upper edge of the cast acrylic panel, creating a smooth initial flow. Water flow rates were measured via a flow meter (Micro Motion ${ }^{\circledR} 3700$ MVD). Regular heated tap water $\left(43^{\circ} \mathrm{C}\right)$ was used for all experiments. All images were taken using an IR camera $(\mathrm{SC} 655,50 \mathrm{~Hz}, 640 \times 480)$. All thermal images were corrected for perspective projection using a 4 point transformation in Matlab. The tubular water applicator and the entire apparatus were installed on a Aluminum frame (80/20 Inc.), which allowed variation of the panel inclination angle between $5^{\circ}$ and $90^{\circ}$.

\section{Results}

The model has previously been validated against the Nusselt solution theory and experimental data for film thickness and velocity of continuous thin film flow over an inclined surface [20]. The results for this work are focused on partially wetted flow behavior.

Experiments and simulations were performed for partially wetted flow conditions over a range of flow rates and inclination angles. A uniform grid spacing of $3.8 \mathrm{~mm}$ was used for the calculations. The simulations are compared here to the experimentally measured partially wetted flow conditions. First, qualitative visual comparison is shown for the flow behavior, followed by comparison based on the measured wetted area fraction.

Figure 1(a-d) shows instantaneous experimental images of flows on a $5^{\circ}$ sloped surface. The wetted surface is displayed as black, while the dry surfaces are shown as white. At the lower flow rates, partial wetting of the surface is observed, yielding a rivulet based flow. As flow rate increases past a critical value, the surface becomes flooded. The transition from rivulet flow to continuous film flow occurs between $\Gamma=108 \mathrm{~g} / \mathrm{m} / \mathrm{s}$ and $\Gamma=174 \mathrm{~g} / \mathrm{m} / \mathrm{s}$. In the flooded condition, considerable 'necking' of the flow is observed. The extent of necking decreases with increasing flow rate.

Simulation results for the $5^{\circ}$ incline condition are shown in Figure 1(e-h). The model shows considerably less meandering behavior for the rivulet flow. Still, similarities are seen in the wetting behavior of the liquid film. The lower flow rate simulations predict rivulet flow, while the higher flow rate simulations 


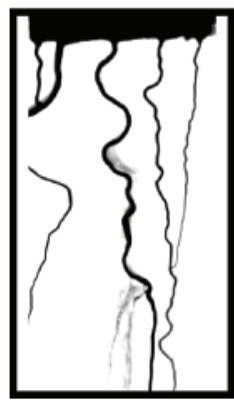

(a) $\Gamma=31 \mathrm{~g} / \mathrm{m} / \mathrm{s}$

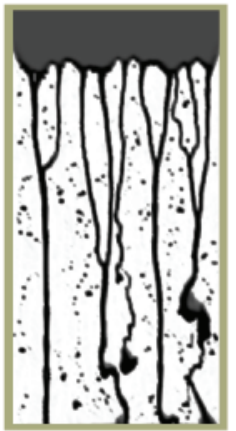

(e) $\Gamma=50 \mathrm{~g} / \mathrm{m} / \mathrm{s}$

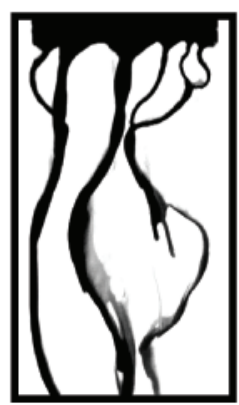

(b) $\Gamma=108 \mathrm{~g} / \mathrm{m} / \mathrm{s}$

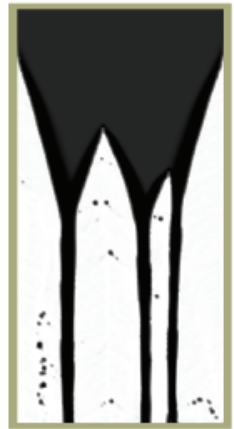

(f) $\Gamma=150 \mathrm{~g} / \mathrm{m} / \mathrm{s}$

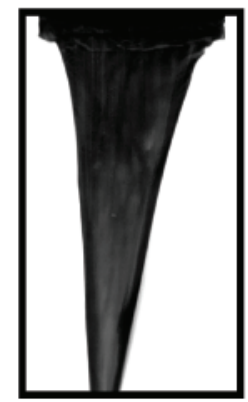

(c) $\Gamma=174 \mathrm{~g} / \mathrm{m} / \mathrm{s}$

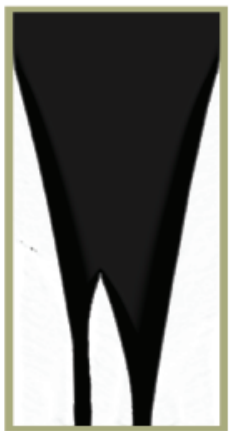

(g) $\Gamma=200 \mathrm{~g} / \mathrm{m} / \mathrm{s}$

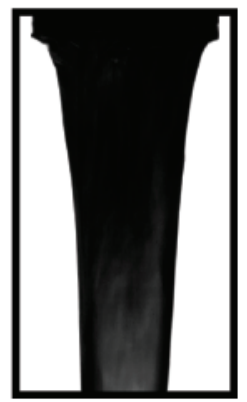

(d) $\Gamma=309 \mathrm{~g} / \mathrm{m} / \mathrm{s}$

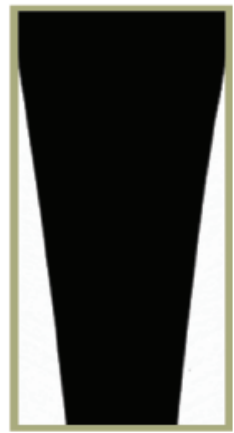

(h) $\Gamma=400 \mathrm{~g} / \mathrm{m} / \mathrm{s}$

Figure 1: Experimental film flow (a-d) compared with simulated film flow (e-h) ( $5^{\circ}$ incline, $\beta=0.4$ ).

predict flooded conditions. Even the general trend of the reduction in necking as flow rate increases is captured in the model, if only qualitatively. The simulation results show isolated droplets that form as a rivulet passes. This behavior was observed experimentally also, however the post-processing of the experimental images removed the droplets from visibility.

Figure 2(a-d) shows experimental images of flows on a $90^{\circ}$ sloped surface for a range of flow rates. The transition from rivulet flow to flooded film flow occurs between $\Gamma$ values of 125 and $212 \mathrm{~g} / \mathrm{m} / \mathrm{s}$. Simulation results for the $90^{\circ}$ incline condition are shown in Figure 2(e-h). The transition from partially wetted flow to flooded flow occurs between $\Gamma=150 \mathrm{~g} / \mathrm{m} / \mathrm{s}$ to $\Gamma=300 \mathrm{~g} / \mathrm{m} / \mathrm{s}$.

Several possible causes exist for the discrepancies seen between the qualitative visual comparisons of the experimental images and simulated results. First, the simulations used a steady inlet condition, with film thickness and velocity introduced at the top of the panel uniformly in space and time. No perturbations of the flow were simulated at the inlet. This behavior differs from the experimentally observed fluctuations in film thickness at the inlet. Another cause for the discrepancy is possibly the thin film assumption. The simulations do not account 


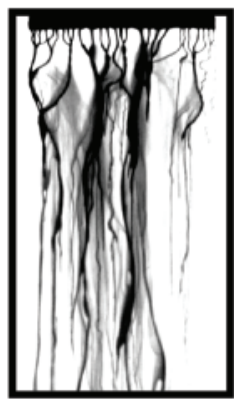

(a) $\Gamma=73 \mathrm{~g} / \mathrm{m} / \mathrm{s}$

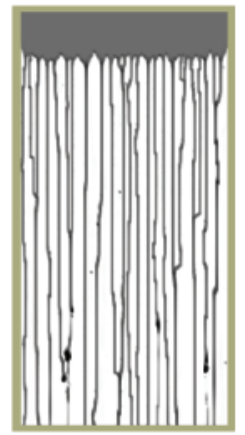

(e) $\Gamma=50 \mathrm{~g} / \mathrm{m} / \mathrm{s}$

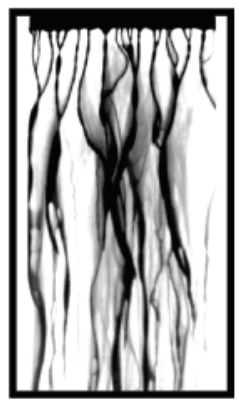

(b) $\Gamma=125 \mathrm{~g} / \mathrm{m} / \mathrm{s}$

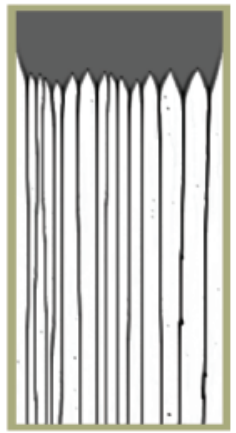

(f) $\Gamma=150 \mathrm{~g} / \mathrm{m} / \mathrm{s}$

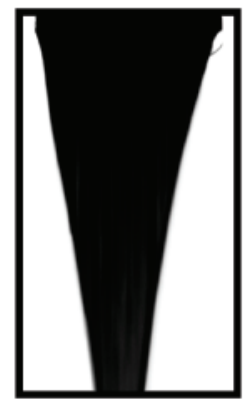

(c) $\Gamma=212 \mathrm{~g} / \mathrm{m} / \mathrm{s}$

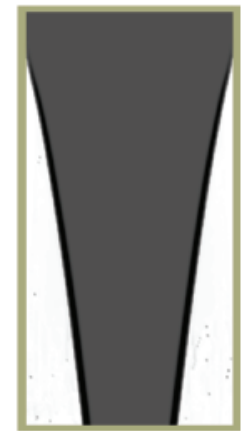

(g) $\Gamma=300 \mathrm{~g} / \mathrm{m} / \mathrm{s}$

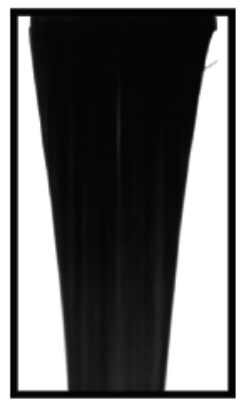

(d) $\Gamma=505 \mathrm{~g} / \mathrm{m} / \mathrm{s}$

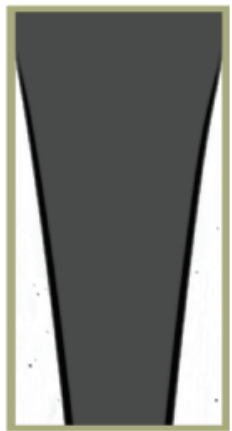

(h) $\Gamma=400 \mathrm{~g} / \mathrm{m} / \mathrm{s}$

Figure 2: Experimental film flow (a-d) compared with simulated film flow (e-h) $\left(90^{\circ}\right.$ incline, $\left.\beta=1.0\right)$.

for any surface-normal components of the flow. Experimentally, observations show that such surface-normal velocities do seem to occur. Another possible discrepancy is the assumed viscous shear stress that is based on a quadratic velocity profile. This assumption holds for continuous films, but may break down when the flow is constrained to narrow rivulets. Also, local variations in surface roughness and/or curvature of the panel are not captured in the simulations. Finally, thermocapillary instabilities may play a role in determining the flow characteristics. The experimental flow was set to a temperature higher than that of the panel to assist in image acquisition with an IR camera. Although cast acrylic is not a very conductive material $(k=0.56 \mathrm{~W} / \mathrm{m}-\mathrm{K})$, small thermal gradients may give rise to thermocapillary instabilities and enhance the meandering behavior of the flow. Experimental observation has shown markedly different behavior for a hot film on a cold panel versus a cold film on a hot panel [21,22].

A more quantitative comparison was made between the experimental and simulated results. The wetted area fraction (WAF) is the fraction of the total solid panel surface area that is covered by liquid. The WAF was digitally extracted from 


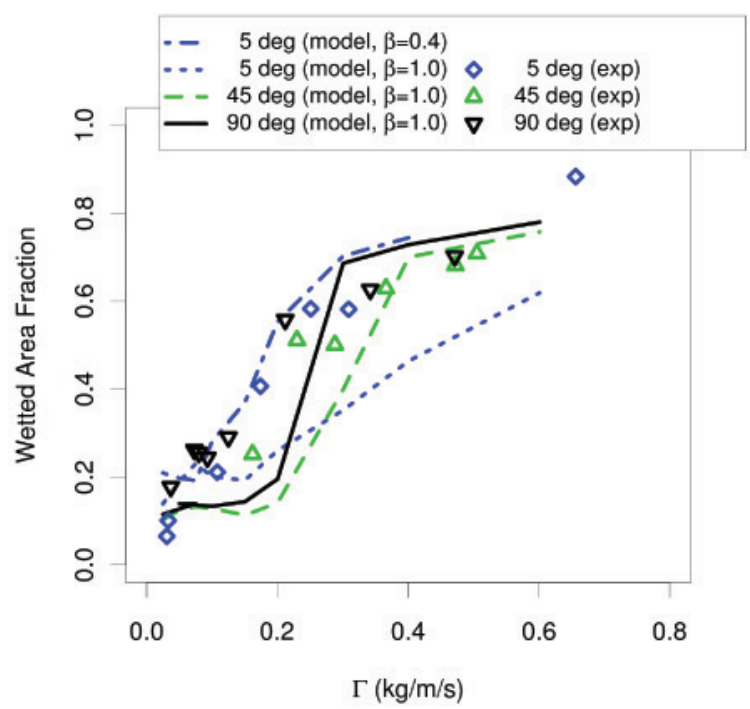

Figure 3: Wetted area fraction as a function of flow rate.

the experimental results using a threshold method in Matlab. Figure 3 shows this comparison to the fractions computed from the simulation results.

At the lower flow rates (rivulet flow), the wetted area fraction is between 0.1 and 0.2 for both model and experiments. As the flow rate increases, the model predicts a slightly more rapid transition to flooded film flow for the $45^{\circ}$ and $90^{\circ}$ film cases. The $5^{\circ}$ incline results demonstrate a much more gradual transition to fully flooded conditions. A simulated result for the $5^{\circ}$ incline case with a value of $\beta=0.4$ is shown in Figure 3 for comparison.

\section{Summary and conclusions}

The partial wetting behavior of thin-film flow was simulated for a wide range of flow rates and for three inclination angles. Although a few of the more subtle behaviors of the flow are not captured (e.g. meandering rivulets), the visual comparisons to the experimental flow patterns are encouraging. In particular, the rivulet flow transitioning to flooded film flow behavior of the model is very reasonable. The predictions for wetted area fraction are also encouraging. The wetted area fraction predictions were shown to be sensitive to the $\beta$ parameter. However, values for $\beta=1$ yielded a good match to the experimental values for the $45^{\circ}$ and $90^{\circ}$ cases.

More work is needed in simulating realistic inlet boundary conditions for the film. Ideally the inlet flow rate would be allowed to vary as a function of space and time. Also, the sensitivity of the simulation results to contact angle and grid spacing needs to be studied. 


\section{Acknowledgements}

This work was funded by FM Global as part of the Strategic Research Program for Fire and Suppression Modeling. OpenCFD Ltd is gratefully acknowledged for the development of the underlying framework for the thin film solver. OpenFOAM is a registered trademark of OpenCFD Limited, the producer of the OpenFOAM software.

\section{References}

[1] Hopf, L., Turbulenz bei einem flusse. Annalen der Physik, 337(9), pp. 777808, 1910.

[2] Nusselt, W., Die oberflachenkondensation des wasserdampfes. Zeitschrift des Vereines Deutscher Ingenieure, 60, pp. 541-546 and 569-575, 1916.

[3] Nusselt, W., Heat transfer in the trickle cooler. Zeitschrift des Vereines Deutscher Ingenieure, 67, pp. 206-210, 1923.

[4] Fulford, G.D., The flow of liquids in thin films. Academic Press, volume 5 of Advances in Chemical Engineering, pp. 151-236, 1964.

[5] Tong, A.Y. \& Wang, Z., A numerical method for capillarity-dominant free surface flows. Journal of Computational Physics, 221(2), pp. 506 - 523, 2007.

[6] Saha, A.A. \& Mitra, S.K., Effect of dynamic contact angle in a volume of fluid (vof) model for a microfluidic capillary flow. Journal of Colloid and Interface Science, 39(2), pp. 461-480, 2009.

[7] Diez, J.A. \& Kondic, L., Computing three-dimensional thin film flows including contact lines. Journal of Computational Physics, 183(1), pp. 274306, 2002.

[8] Frassy, J., Lecot, C., Murariu, M., Delattre, C. \& Soucemarianadin, A., Transient spreading of films on heterogeneous surfaces. Proceedings of the COMSOL Users Conference 2007 Grenoble, 2007.

[9] King, A.A., Cummings, L.J., Naire, S. \& Jensen, O.E., Liquid film dynamics in horizontal and tilted tubes: dry spots and sliding drops. Physics of Fluids, 2007.

[10] Bai, C.X. \& Gosman, A.D., Mathematical modeling of wall films formed by impinging sprays. Society of Automotive Engineers, (SAE 960626), 1996.

[11] Stanton, D. \& Rutland, C., Multi-dimensional modeling of heat and mass transfer of fuel films resulting from impinging sprays. SAE Technical Paper Series, 1998.

[12] O'Rourke, P.J. \& Amsden, A.A., A particle numerical model for wall film dynamics in port-fuel injected engines. SAE Paper 961961, 1996.

[13] O'Brien, S. \& Schwartz, L., Theory and modeling of thin film flows. Encyclopedia of Surface and Colloid Science, p. 5283, 2002.

[14] Landau, L.D. \& Lifshitz, E.M., Fluid Mechanics, Second Edition: Volume 6 (Course of Theoretical Physics), 1984. 
[15] Trela, M., A semi-theoretical model of stability of vertical falling liquid films. Chemical Engineering Science, 49(7), pp. 1007 - 1013, 1994.

[16] Hartley, D. \& Murgatroyd, W., Criteria for the break-up of thin liquid layers flowing isothermally over solid surfaces. International Journal of Heat and Mass Transfer, 7(9), pp. 1003 - 1015, 1964.

[17] de Gennes, P.G., Borochard-Wyart, F. \& Quere, D., Capillarity and Wetting Phenomena. Springer, 2003.

[18] Semiczek-Szulc, S. \& Mikielewicz, J., Experimental investigation of contact angles of rivulets flowing down a vertical solid surface. International Journal of Heat and Mass Transfer, 21(12), pp. 1625 - 1625, 1978.

[19] Ponter, A.B. \& Aswald, K.M., Minimum thickness of a liquid film flowing down a vertical surface-validity of Mikielewicz and Moszynskfs equation. International Journal of Heat and Mass Transfer, 20(5), pp. 575 - 576, 1977.

[20] Meredith, K.V., Xin, Y.B. \& de Vries, J., A numerical model for simulation of thin-film water transport over solid fuel surfaces. Fire Safety Science, submitted, 2011.

[21] Zhang, F., Zhao, X., Geng, J., Wu, Y.T. \& Zhang, Z., A new insight into Marangoni effect in non-isothermal falling liquid films. Experimental Thermal and Fluid Science, 31, pp. 361-365, 2007.

[22] Zhang, F., Wu, Y.T., Geng, J. \& Zhang, Z.B., An investigation of falling liquid films on a vertical heated/cooled plate. International Journal of Multiphase Flow, 34(1), pp. 13 - 28, 2008. 